\title{
Solution of the Generalized Abel Integral Equation by Using Almost Bernstein Operational Matrix
}

\author{
Sandeep Dixit ${ }^{1}$, Rajesh K. Pandey ${ }^{2}$, Sunil Kumar ${ }^{1}$, Om P. Singh ${ }^{1}$ \\ ${ }^{1}$ Department of Applied Mathematics, Institute of Technology, Banaras Hindu University, Varanasi, India \\ ${ }^{2}$ PDPM Indian Institute of Information Technology, Design and Manufacturing, Jabalpur, India \\ E-mail: \{singhom, skiitbhu\}@gmail.com, \{opsingh.apm, sdixit.rs.apm\}@itbhu.ac.in,rkpandeyy@iiitdmj.ac.in \\ Received June 12, 2011; revised July 5, 2011; accepted July 15, 2011
}

\begin{abstract}
A direct almost Bernstein operational matrix of integration is used to propose a stable algorithm for numerical inversion of the generalized Abel integral equation. The applicability of the earlier proposed methods was restricted to the numerical inversion of a part of the generalized Abel integral equation. The method is quite accurate and stable as illustrated by applying it to intensity data with and without random noise to invert and compare it with the known analytical inverse. Thus it is a good method for applying to experimental intensities distorted by noise.
\end{abstract}

Keywords: Abel Inversion, Bernstein Polynomials, Almost Bernstein Operational Matrix of Integration, Noise Resistance

\section{Introduction}

Abel's integral equation [1] occurs in many branches of science. Usually, physical quantities accessible to measurement are quite often related to physically important but experimentally inaccessible ones by Abel's integral equation. Some of the examples are: microscopy [2], seismology [3,4], radio astronomy [5], satellite photometry of airglows [6], electron emission [7], atomic scattering [8], radar ranging [9], and optical fiber evaluation [10-12]. But it is most extensively used in flame and plasma diagnostics [13-15] and X-ray radiography [16-19].

Recently, Chakrabarti [20] employed a direct function theoretic method to determine the closed form solution of the following generalized Abel integral equation

$$
\begin{aligned}
& a(x) \int_{\alpha}^{x} \frac{\phi(t) \mathrm{d} t}{(x-t)^{\mu}}+b(x) \int_{x}^{\beta} \frac{\phi(t) \mathrm{d} t}{(t-x)^{\mu}}=f(x), \\
& (0<\mu<1),(\alpha \leq x \leq \beta),
\end{aligned}
$$

where the coefficients $a(x)$ and $b(x)$ do not vanish simultaneously.

Earlier the generalized Abel equation (1) was examined in Gakhov's book [21], under the special assumptions that the coefficients $a(x)$ and $b(x)$ satisfy Holder's condition in $[\alpha, \beta]$, whereas the forcing term $f(x)$ and the unknown function $\phi(x)$ belong to those class of functions which admit representations of the form

$$
\left.\begin{array}{l}
f(x)=[(x-\alpha)(\beta-x)]^{\varepsilon} f^{*}(x), \\
\phi(x)=\frac{\phi^{*}(x)}{[(x-\alpha)(\beta-x)]^{1-\mu-\varepsilon}}
\end{array}\right\}(\varepsilon>0),
$$

where $f^{*}(x)$ possesses a Holder continuous derivative in $[\alpha, \beta]$ and $\phi^{*}(x)$ satisfies Holder's condition in $[\alpha, \beta]$.

The method of Gakhov's has a particular disadvantage in the sense that while solving a singular equation that involves integrals only with weak singularity of the type $(t-x)^{-\mu}(0<\mu<1)$, occurrence of strongly singular integrals involving Cauchy type singularities of the type $(t-x)^{-1}$ has to be permitted [20,21]. Chakrabarti [20] obtained the solution involving only weakly singular integrals of the Abel type and thus Cauchy type singular integrals were avoided. But the numerical inversion is still needed for its application in physical models since the experimental data for the intensity $f(x)$ is available only at a discrete set of points and it may also be distorted by noise.

The aim of the present paper is to propose a new stable algorithm for the numerical inversion of Abel's integral equation (1), based on the newly constructed almost Bernstein operational matrices of integration. Numerical examples are given to illustrate the accuracy and stability 
of the proposed algorithm.

\section{The Bernstein Polynomials}

A Bernstein polynomial, named after Sergei Natanovich Bernstein, is a polynomial in the Bernstein form that is a linear combination of Bernstein basis polynomials.

The Bernstein basis polynomials of degree $n$ are defined by

$$
B_{i, n}(t)=\left(\begin{array}{c}
n \\
i
\end{array}\right) t^{i}(1-t)^{n-i}, \forall i=0,1,2, \cdots, n .
$$

There are $(n+1) n^{\text {th }}$ degree Bernstein basis polynomials forming a basis for the linear space $V_{n}$ consisting of all polynomials of degree less than or equal to $n$ in $\mathbf{R}[\mathrm{x}]$ - the ring of polynomials over the field $\mathbf{R}$. For mathematical convenience, we usually set $B_{i, n}=0$ if $i<0$ or $i>n$.

Any polynomial $B(x)$ in $V_{n}$ may be written as

$$
B(x)=\sum_{i=0}^{n} \beta_{i} B_{i, n}(x) .
$$

Then $B(x)$ is called a polynomial in Bernstein form or Bernstein polynomial of degree $n$. The coefficients $\beta_{i}$ are called Bernstein or Bezier coefficients. But several mathematicians call Bernstein basis polynomials $B_{i, n}(x)$ as the Bernstein polynomials. We will follow this convention as well. These polynomials have the following properties:

1) $B_{i, n}(0)=\delta_{i 0}$ and $B_{i, n}(1)=\delta_{i n}$, where $\delta$ is the Kronecker delta function.

2) $B_{i, n}(t)$ has one root, each of multiplicity $i$ and $n-i$, at $t=0$ and $t=1$ respectively.

3) $B_{i, n}(t) \geq 0$ for $t \in[0,1]$ and

$$
B_{i, n}(1-t)=B_{n-i, n}(t) \text {. }
$$

4) For $i \neq 0, B_{i, n}$ has a unique local maximum in $[0,1]$ at $t=i / n$ and the maximum value

$$
i^{i} n^{-n}(n-i)^{n-i}\left(\begin{array}{l}
n \\
i
\end{array}\right) \text {. }
$$

5) The Bernstein polynomials form a partition of unity i.e. $\sum_{i=0}^{n} B_{i, n}(t)=1$.

Using Gram-Schmidt orthonormalization process on $B_{i, n}$, we obtain a class of orthonormal polynomials. We call them orthonormal Bernstein polynomials of order $n$ and denote them by $b_{0 n}, b_{1 n}, \cdots, b_{n n}$.

\section{Function Approximation}

A function $f \in L^{2}[0,1]$ may be written as

$$
f(t)=\lim _{n \rightarrow \infty} \sum_{i=0}^{n} c_{i n} b_{i n}(t),
$$

where, $c_{i n}=\left\langle c, b_{i n}\right\rangle$ and $\langle$,$\rangle is the standard inner prod-$ uct on $L^{2}[0,1]$.

If the series (5) is truncated at $n=m$, then we have

$$
f \cong \sum_{i=0}^{m} c_{i m} b_{i m}=C^{T} B(t),
$$

where, $C$ and $B(t)$ are $(m+1) \times 1$ matrices given by

$$
C=\left[c_{0 m}, c_{1 m}, \cdots, c_{m m}\right]^{T},
$$

and

$$
B(t)=\left[b_{0 m}(t), b_{1 m}(t), \cdots, b_{m m}(t)\right]^{T} .
$$

\section{Solution of Generalized Abel Integral Equation}

In this section we solve generalized Abel integral equation by orthonormal Bernstein polynomials.

Using Equation (8), we approximate $\phi(x)$ and $f(x)$ as

$$
\tilde{\phi}(x)=C^{T} B(x), \tilde{f}(x)=F^{T} B(x),
$$

where the matrix $F$ is known. Then from equation (1) and (9) we have

$$
a(x) \int_{\alpha}^{x} \frac{C^{T} B(t) \mathrm{d} t}{(t-x)^{\mu}}+b(x) \int_{x}^{\beta} \frac{C^{T} B(t) \mathrm{d} t}{(t-x)^{\mu}}=F^{T} B(x)
$$

From Equation (8) and from the derived formulae,

$$
\begin{aligned}
& \int_{\alpha}^{x} \frac{t^{n} \mathrm{~d} t}{(x-t)^{\mu}}=\Gamma(1+n) x^{-\mu} \\
& \cdot\left(\frac{\Gamma(1-\mu) x^{n+1}}{\Gamma(2+n-\mu)}-\alpha^{n+1}{ }_{2} \tilde{F}_{1}\left(n+1, \mu ; n+2 ; \frac{\alpha}{x}\right)\right),
\end{aligned}
$$

$\int_{x}^{\beta} \frac{t^{n} \mathrm{~d} t}{(t-x)^{\mu}}=\Gamma(-n+\mu+1)$

$\cdot\left(\frac{\Gamma(1-\mu) x^{1+n-\mu}}{\Gamma(-n)}-\beta^{-\mu+n+1}{ }_{2} \tilde{F}_{1}\left(\mu,-n+\mu-1 ; \mu-n ; \frac{x}{\beta}\right)\right)$,

it is obvious that

$$
\int_{\alpha}^{x} \frac{B(t)}{(x-t)^{\mu}} \mathrm{d} t=P B(x), \int_{x}^{\beta} \frac{B(t)}{(t-x)^{\mu}} \mathrm{d} t=Q(x),
$$

where $P$ and $Q$ are $(m+1) \times(m+1)$ matrices, which we call as almost Bernstein operational matrix of integration for Abel integral equation with generalized kernel.

Substituting (13) in (10), we get

$$
C^{T}=F^{T}(a(x) P+b(x) Q)^{-1}
$$


Hence, the approximate solution $\tilde{\phi}(t)$ for generalized Abel integral equation (1) is obtained by putting the value of $C^{T}$ from (14) in (9).

\section{Illustrative Examples}

The following examples are solved with and without noise terms to illustrate the efficiency and stability of our method. Note that in all the examples to follow, the series (5) is truncated at level $m=6$ and hence the almost operational matrix in (13) is of order $7 \times 7$.

The accuracy of the proposed algorithm is demonstrated by calculating the parameters of absolute error $\Delta \phi\left(t_{i}\right)$ and average deviation $\sigma$ also known as root mean square error (RMS). They are calculated using the following equations:

$$
E\left(t_{i}\right)=\left|\phi\left(t_{i}\right)-\tilde{\phi}\left(t_{i}\right)\right|
$$

and

$$
\begin{aligned}
\sigma_{N} & =\left\{\frac{1}{N} \sum_{i=1}^{N}\left[\phi\left(t_{i}\right)-\tilde{\phi}\left(t_{i}\right)\right]^{2}\right\}^{1 / 2}=\left\{\frac{1}{N} \sum_{i=1}^{N} \Delta \phi^{2}\left(t_{i}\right)\right\}^{1 / 2} \\
& =\|\phi\|_{2},
\end{aligned}
$$

where $\tilde{\phi}\left(t_{i}\right)$ is the approximate solution calculated at point $t_{i}$ and $\left(t_{i}\right)$ is the exact solution at the corresponding point. Note that $\sigma$, henceforth, denoted by $\sigma_{N}$ (for computational convenience) is the discrete $l^{2}$-norm of the absolute error $\Delta \phi$ denoted by $\|\Delta \phi\|_{2}$.

Note that the calculation of $\sigma_{N}$ is performed by taking $N=1000,500$ in Equation (15). In all the examples, the exact and noisy profiles are denoted by $f(x)$ and $f^{\delta}(x)$, respectively, where $f^{\delta}(x)$ is obtained by adding a noise $\delta$ to $f(x)$ such that

$f^{\delta}\left(x_{i}\right)=f\left(x_{i}\right)+\delta \theta_{i}$, where $x_{i}=i h, i=1, \cdots, N, N h=1$ and $\theta_{i}$ is the uniform random variable with values in $[-1,1]$ such that $\max _{1 \leq i \leq N}\left|f_{i}^{\delta}(x)-f_{i}(x)\right| \leq \delta$.

The following examples are solved with and without noise to illustrate the efficiency and stability of our method by choosing two different values of the noises $\delta_{j}$ as $\delta_{0}=0, \delta_{1}=\sigma_{N}$. In all the examples to follow, we take four different values of $\mu$ as $1 / 10,1 / 4,1 / 2,3 / 4$ and compute the corresponding errors $E(t)$ and level them as $E 1(t), E 2(t), E 3(t), E 4(t)$ respectively. Then a noise term $\delta_{1}$ is introduced in forcing term $f(x)$ and for $N=1000,500$ the corresponding errors $E 5(t)$, $E 6(t), E 7(t), E 8(t)$ and $E 9(t), E 10(t), E 11(t), E 12(t)$, are computed for the four chosen values of $\mu$ as mentioned above. In all the figures some of the error terms $E_{j}(t)$ are multiplied by 10 or some power of 10 for suitable scaling. Also we tabulated the approximate and exact solutions through Tables 1-4 for the four examples given below for various values of $t$.

Example 1. Consider the generalized Abel integral equation with $a(x)=b(x)=1$ and

$$
\begin{aligned}
& f(x)= \\
& \frac{10395 \sqrt{\pi} x^{6-\mu} \Gamma(1-\mu)\left((1 / x)^{\mu} x^{\mu+1 / 2}-\sqrt{x} \sec (\pi \mu)\right)}{65 \Gamma\left(\frac{15}{2}-\mu\right)} \\
& -\Gamma\left(\mu-\frac{13}{2}\right)_{2} \tilde{F}_{1}\left(\mu-\frac{13}{2}, \mu, \mu-\frac{11}{2}, x\right),
\end{aligned}
$$

where ${ }_{2} \tilde{F}_{1}$ is the regularized hypergeometric function. This has the exact solution $\phi(t)=t^{1 / 2}$.

Figure 1 illustrates the effect of the absolute errors without noise for different values of $\mu$, whereas Figures 2 and $\mathbf{3}$ show the absolute errors with noise term $\delta_{1}$ added to the forcing term $f(x)$ for $N=1000$, 500 respectively. Table 1 compares the approximate and exact values of Example 1.

Example 2. Consider the generalized Abel integral equation with $a(x)=b(x)=1$ and

$$
\begin{aligned}
f(x)= & \frac{2(1-x)^{2-\mu}\left(3(1+x)^{2}-(4+3 x) \mu+\mu^{2}\right)}{(\mu-4)(\mu-3)(\mu-2)(\mu-1)} \\
& +\frac{x^{-\mu}\left(x^{2}-\frac{6 x^{4}}{12-7 \mu+\mu^{2}}\right)}{2+(\mu-3) \mu}
\end{aligned},
$$

having the exact solution $\phi(t)=t-t^{3}$.

Figure 4 illustrates the effect of the absolute errors

Table 1. Approximate and exact solution of Example 1.

\begin{tabular}{ccccccc}
\hline $\mathrm{t}$ & 0.0 & 0.2 & 0.4 & 0.6 & 0.8 & 1.0 \\
\hline$\phi(t)$ & 0 & 0.00014 & 0.00648 & 0.06023 & 0.29309 & 1.0000 \\
$\phi(t)$ at $\mu=1 / 10$ & 0.00013 & 0.00018 & 0.00647 & 0.06020 & 0.29308 & 1.00025 \\
$\phi(t)$ at $\mu=1 / 4$ & 0.00001 & 0.00016 & 0.00646 & 0.06020 & 0.29311 & 1.00003 \\
$\phi(t)$ at $\mu=1 / 2$ & -0.00004 & 0.00013 & 0.00646 & 0.06024 & 0.29309 & 1.00004 \\
$\phi(t)$ at $\mu=3 / 4$ & -0.00004 & 0.00011 & 0.00646 & 0.06023 & 0.29308 & 1.00001 \\
\hline
\end{tabular}


Table 2. Approximate and exact solution of Example 2.

\begin{tabular}{ccccccc}
\hline $\mathrm{t}$ & 0.0 & 0.2 & 0.4 & 0.6 & 0.8 & 1.0 \\
\hline$\phi(t)$ & 0 & 0.19200 & 0.33600 & 0.38400 & 0.28800 & 0.0000 \\
$\phi(t)$ at $\mu=1 / 10$ & -0.00001 & 0.19199 & 0.33599 & 0.38400 & 0.28798 & -0.000005 \\
$\phi(t)$ at $\mu=1 / 4$ & -0.00001 & 0.19200 & 0.33601 & 0.38400 & 0.28801 & -0.000033 \\
$\phi(t)$ at $\mu=1 / 2$ & -0.00004 & 0.19200 & 0.33600 & 0.38400 & 0.28800 & -0.000038 \\
$\phi(t)$ at $\mu=3 / 4$ & -0.00037 & 0.19195 & 0.33601 & 0.38396 & 0.28800 & 0.00014 \\
\hline
\end{tabular}

Table 3. Approximate and exact solutions of Example 3.

\begin{tabular}{ccccccc}
\hline $\mathrm{t}$ & 0.0 & 0.2 & 0.4 & 0.6 & 0.8 & 1.0 \\
\hline$\phi(t)$ & 1 & 1.2214 & 1.49182 & 1.82212 & 2.22554 & 2.71828 \\
$\phi(t)$ at $\mu=1 / 10$ & 1.0002 & 1.22145 & 1.49183 & 1.82210 & 2.22558 & 2.71828 \\
$\phi(t)$ at $\mu=1 / 4$ & 1.00004 & 1.22140 & 1.49182 & 1.82214 & 2.22550 & 2.71853 \\
$\phi(t)$ at $\mu=1 / 2$ & 1.00012 & 1.22140 & 1.49183 & 1.82212 & 2.22556 & 2.71831 \\
$\phi(t)$ at $\mu=3 / 4$ & 1.00156 & 1.22116 & 1.49180 & 1.82190 & 2.22557 & 2.71868 \\
\hline
\end{tabular}

Table 4. Approximate and exact solutions of Example 4.

\begin{tabular}{ccccccc}
\hline $\mathrm{t}$ & 0.0 & 0.2 & 0.4 & 0.6 & 0.8 & 1.0 \\
\hline$\phi(t)$ & 0.0 & 0.03959 & 0.07306 & 0.10206 & 0.12764 & 0.15051 \\
$\phi(t)$ at $\mu=1 / 10$ & 0.00017 & 0.03960 & 0.07305 & 0.10204 & 0.12762 & 0.15073 \\
$\phi(t)$ at $\mu=1 / 4$ & 0.00008 & 0.03955 & 0.07307 & 0.10208 & 0.12762 & 0.15056 \\
$\phi(t)$ at $\mu=1 / 2$ & -0.00006 & 0.03960 & 0.07306 & 0.10206 & 0.12764 & 0.15052 \\
$\phi(t)$ at $\mu=3 / 4$ & 0.00007 & 0.03958 & 0.07306 & 0.10205 & 0.12764 & 0.15055 \\
\hline
\end{tabular}

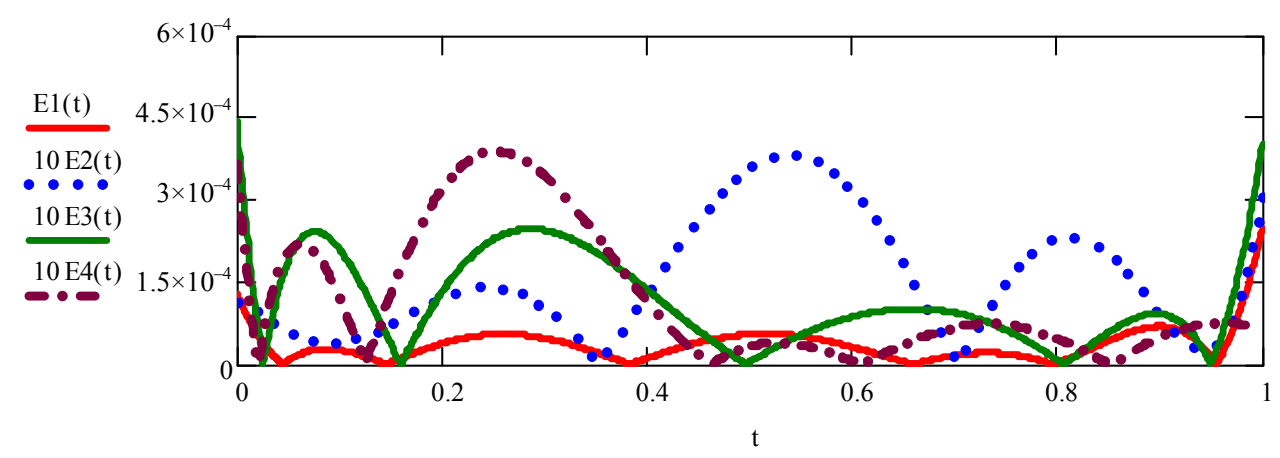

Figure 1. Comparison of absolute errors, Example 1.

without noise for different values of $\mu$, whereas Figures 5 and 6 show the absolute errors with noise term $\delta_{1}$ added to the forcing term $f(x)$ for $N=1000$, 500 respectively. Table 2 compares the approximate and exact values of Example 2.

Example 3. Consider the generalized Abel integral equation with $a(x)=b(x)=1$ and

$$
\begin{aligned}
f(x) & =e^{x}(1-x)^{-\mu}(x-1)^{\mu}(\Gamma(1-\mu, x-1)-\Gamma(1-\mu)) \\
& +e^{x}(\Gamma(1-\mu)-\Gamma(1-\mu, x))
\end{aligned}
$$

This has the exact solution $\phi(t)=e^{t}$.

Figure 7 illustrates the effect of the absolute errors without noise for different values of $\mu$, whereas Figures 8 and 9 show the absolute errors with noise term 


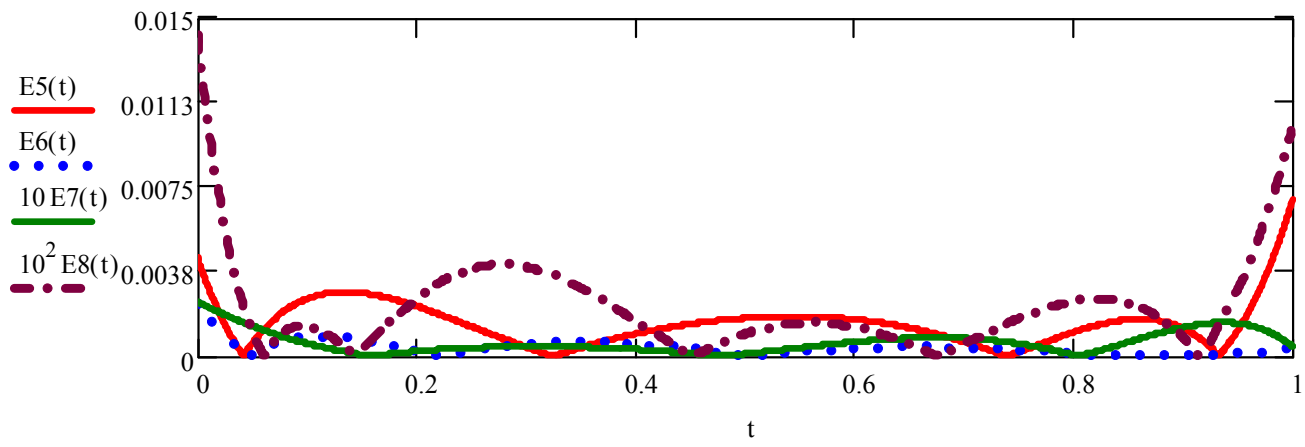

Figure 2. Comparison of absolute errors with noise $\delta_{1}$ for $N=1000$, Example 1 .

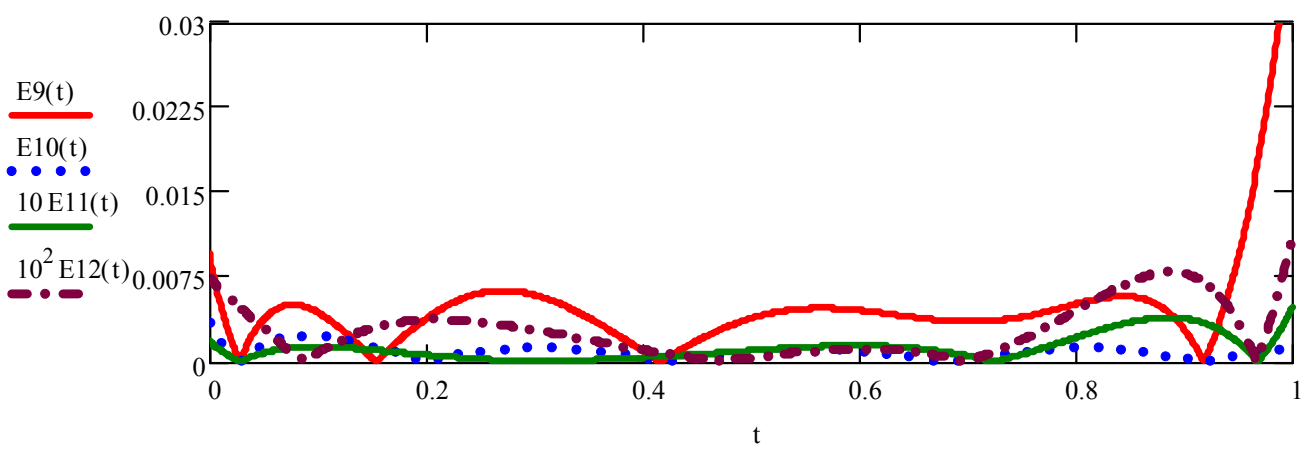

Figure 3. Comparison of absolute errors with noise $\delta_{1}$ for $N=500$, Example 1 .

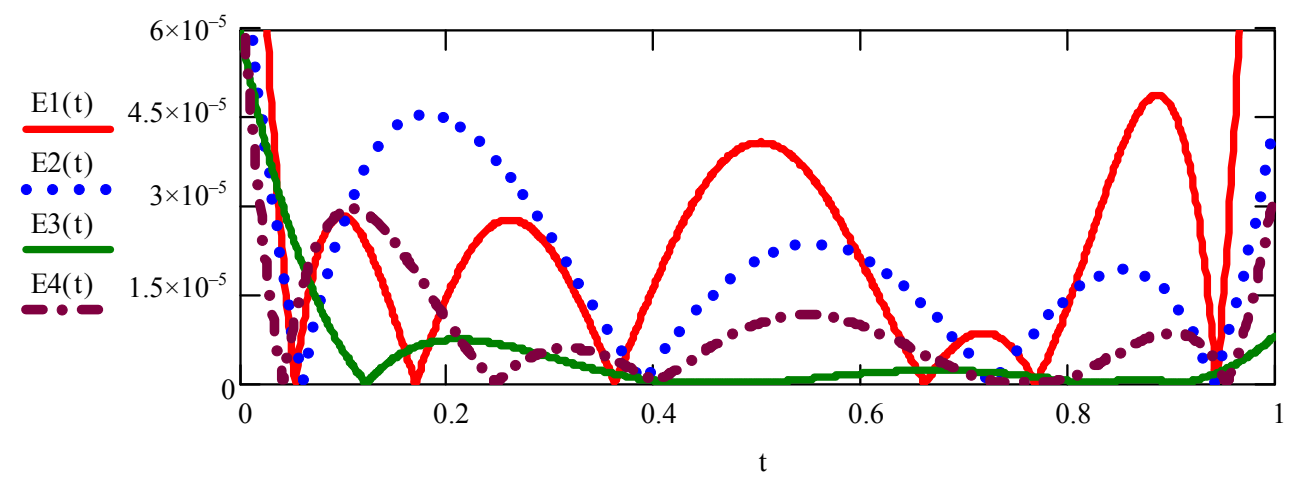

Figure 4. Comparison of absolute errors, Example 2.

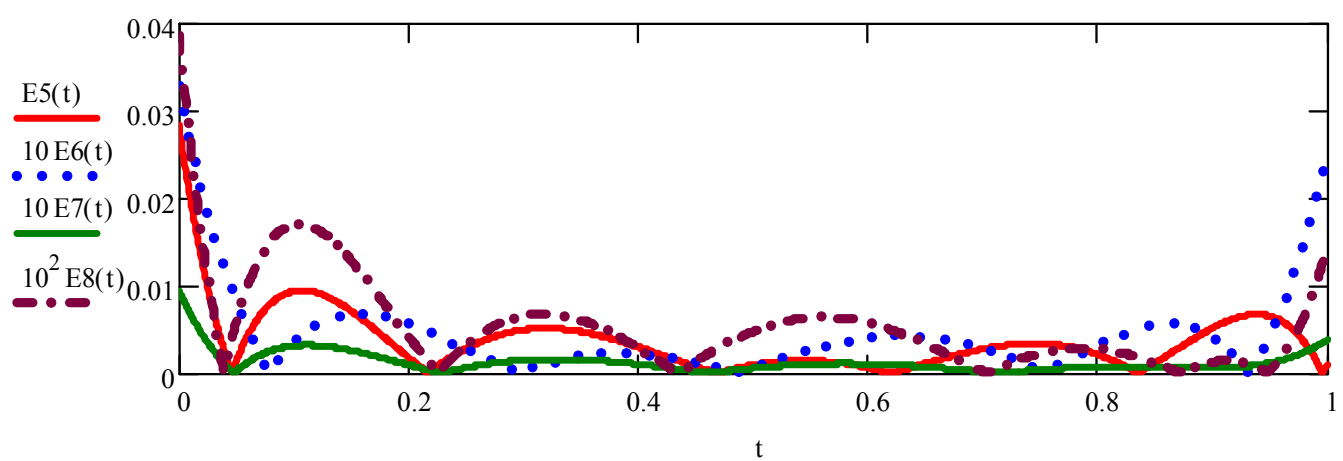

Figure 5. Comparison of absolute errors with noise $\delta_{1}$ for $N=1000$, Example 2 . 


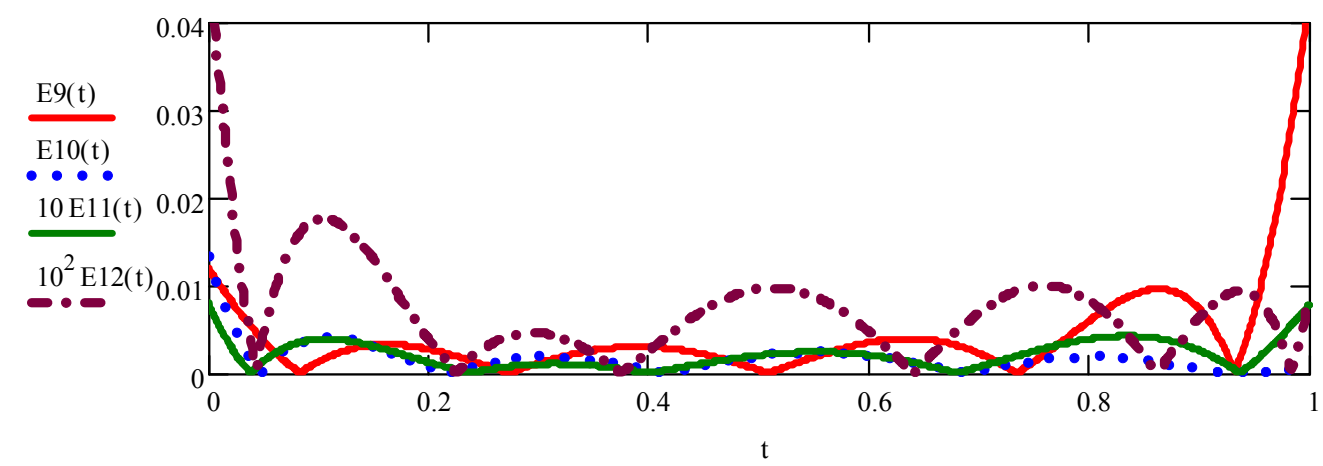

Figure 6. Comparison of absolute errors with noise $\delta_{1}$ for $N=500$, Example 2 .

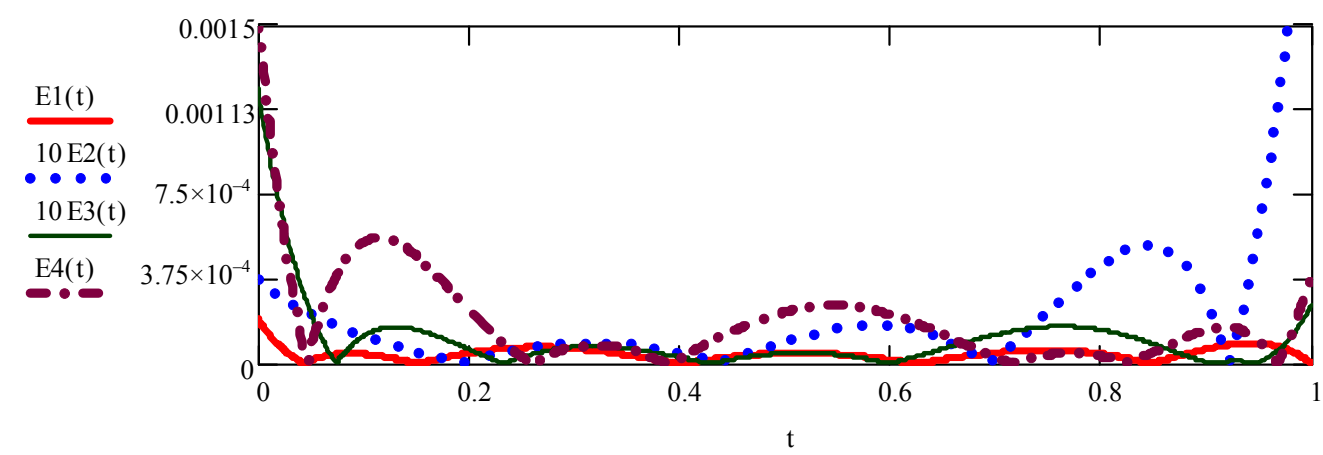

Figure 7. Comparison of absolute errors, Example 3.

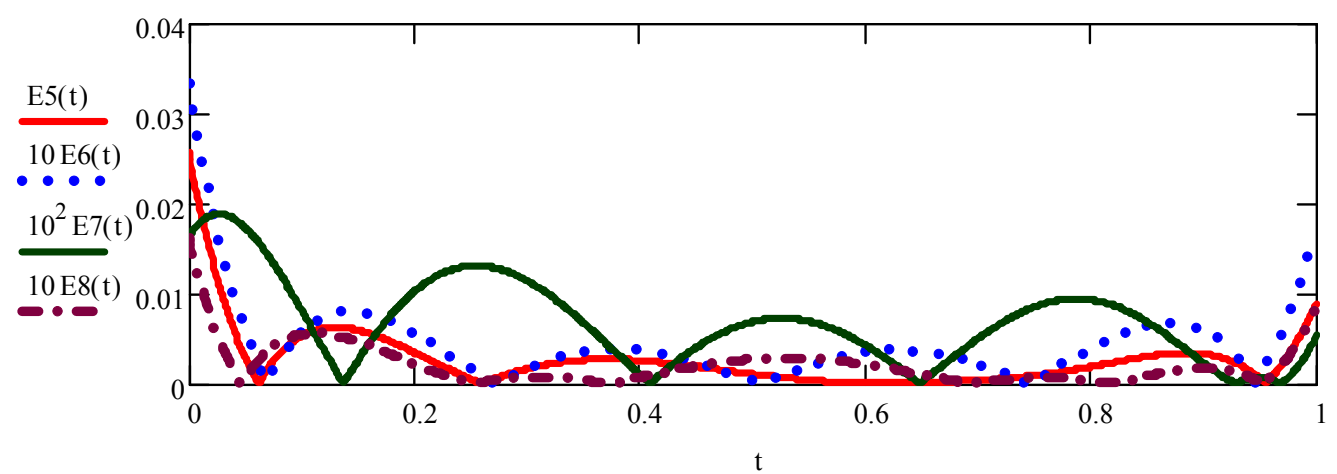

Figure 8. Comparison of absolute errors with noise $\delta_{1}$ for $N=1000$, Example 3 .

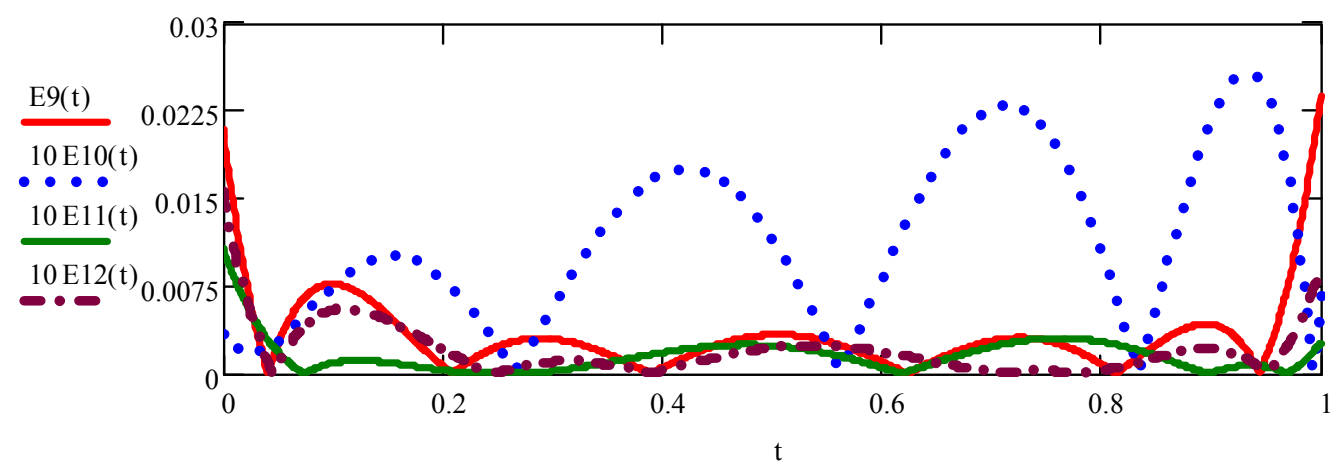

Figure 9. Comparison of absolute errors with noise $\delta_{1}$ for $N=500$, Example 3 . 
$\delta_{1}$ added to the forcing term $f(x)$ for $N=1000$, 500 respectively. Similarly, Table 3 compares the approximate and exact values of Example 3.

Example 4. Now we consider, the following generalized Abel integral equation with $a(x)=b(x)=1$ and (see Equation (19). This has the exact solution $\phi(t)=\log \sqrt{t+1}$.
Figure 10 illustrates the effect of the absolute errors without noise for different values of $\mu$, whereas Figures 11 and 12 show the absolute errors with noise term $\delta_{1}$ added to the forcing term $f(x)$ for $N=1000,500$ respectively. Table 4 compares the approximate and exact values of Example 4.

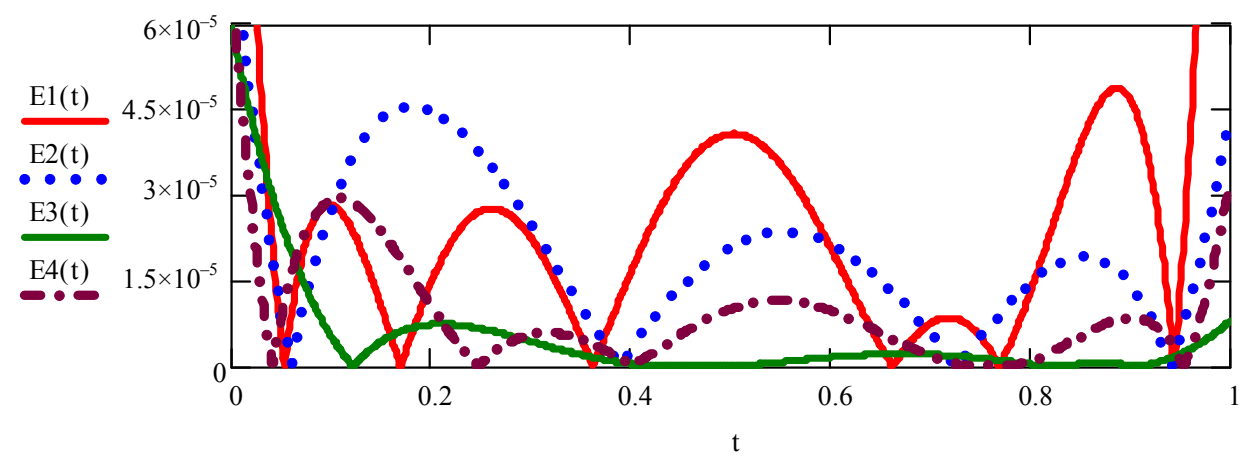

Figure 10. Comparison of absolute errors, Example 4.

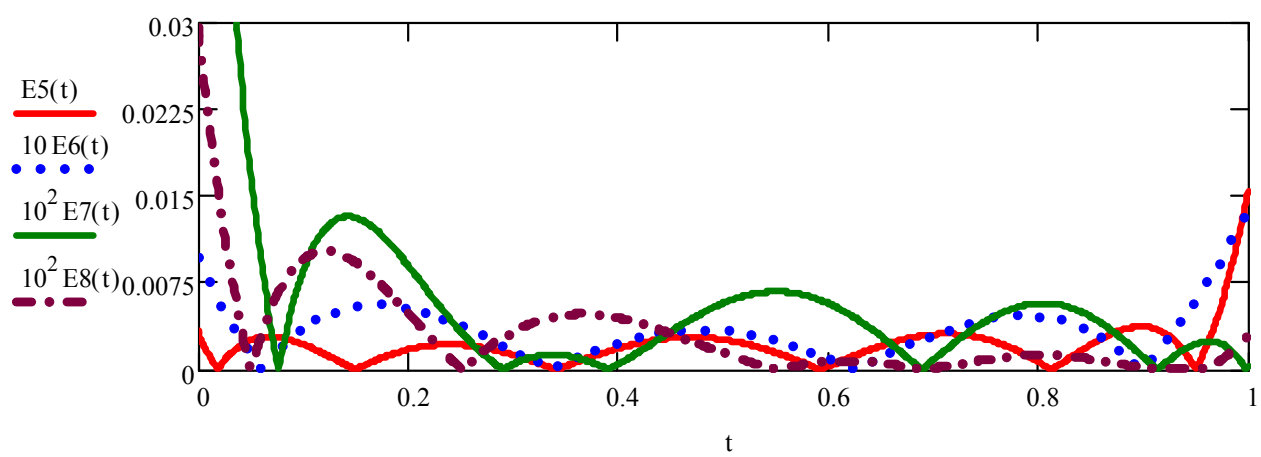

Figure 11. Comparison of absolute errors with noise $\delta_{1}$ for $N=1000$, Example 4 .

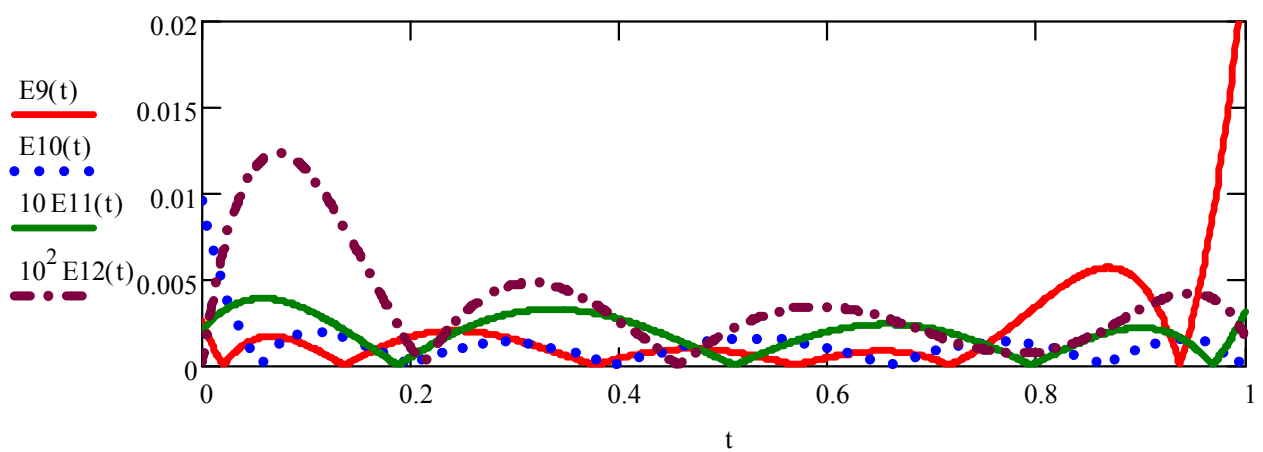

Figure 12. Comparison of absolute errors with noise $\delta_{1}$ for $N=500$, Example 4 .

$$
\begin{array}{r}
f(x)=\frac{\log (2)(1-x)^{1-\mu}}{2-2 \mu}+\frac{1}{2} \Gamma(1-\mu)\left(\left(x(-x)^{1-\mu}+x^{2-\mu}\right)_{2} \tilde{F}_{1}(1,1,3-\mu,-x)\right) \\
+\frac{(-x)^{2-\mu}{ }_{2} \tilde{F}_{1}\left(1,2-\mu, 3-\mu, \frac{x}{x+1}\right)-(1-x)^{2-\mu}{ }_{2} \tilde{F}_{1}\left(1,2-\mu, 3-\mu, \frac{x-1}{x+1}\right)}{x+1}
\end{array}
$$




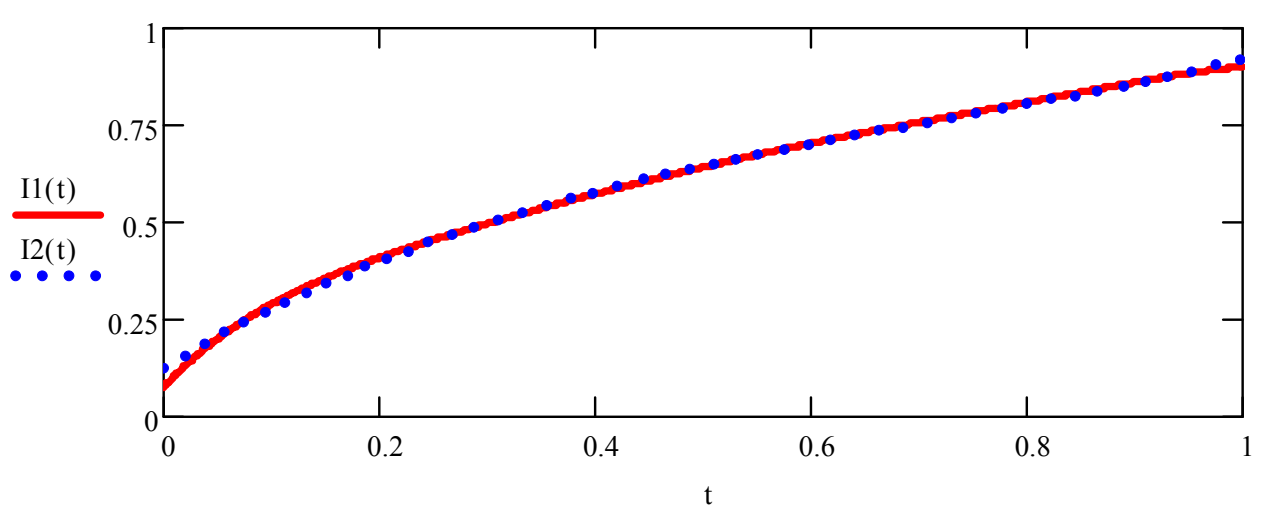

Figure 13. Comparison of approximate solutions, Example 5.

Example 5. Next we consider, the following generalized Abel integral equation with $a(x)=b(x)=1$ and $\mu=1 / 2$.

$$
\begin{aligned}
& f(x)= \\
& \frac{7}{144} x^{3 / 2-\mu}\left(\frac{\mu(\sec (\pi \mu)-1) \Gamma(-\mu)}{\Gamma\left(\frac{5}{2}-\mu\right)}-\frac{2 B_{x}\left(\mu-\frac{3}{2}, 1-\mu\right)}{\sqrt{\pi}}\right),
\end{aligned}
$$

where $B_{x}(a, b)$ is incomplete beta function, defined by $B_{x}(a, b)=\int_{0}^{x} u^{a-1}(1-u)^{b-1} \mathrm{~d} u$.

Figure 13 shows two approximate solutions obtained by applying the operational matrix of integration of order $4 \times 4$ (dotted blue) and the operational matrix of integration of order $7 \times 7$ (solid red). Both the approximate solutions obtained by the two different matrices have similar and almost overlapping evolutions except at the boundary points 0 and 1 . So, we may conclude that the exact solution will have similar evolution.

\section{Conclusions}

We have introduced an almost Bernstein operational matrices of integration to propose a new and stable algorithm for numerical solution of generalized Abel integral equation. It is found that the method is accurate and stable as shown by the numerical examples. Moreover, the algorithm is easy to use since this is a direct method and the solution is obtained by applying the operational matrix of integration directly to the algorithm.

\section{Acknowledgements}

The first author acknowledges the financial support from DST- CIMS, Banaras Hindu University, Varanasi, India under JRF scheme.

\section{References}

[1] N. H. Abel, "Resolution d'un Probleme de Mechanique," Journal für die Reine und Angewandte Mathematik, Vol. 1826, No. 1, 2009, pp. 153-157. doi: $10.1515 /$ crll.1826.1.153

[2] A. J. Jakeman and R. S. Anderssen, "Abel Type Integral Equations in Stereology: I. General Discussion," Journal of Microscopy, Vol. 105, No. 2, 1975, pp. 121-133. doi:10.1111/j.1365-2818.1975.tb04045.x

[3] J. B. Macelwane, "Evidence on the Interior of the Earth Derived from Seismic Sources", In: B. Gutenberg, Ed., Internal Constitution of the Earth, Dover, New York, 1951, pp. 227-304.

[4] S. B. Healy, J. Haase and O. Lesne, "Abel Transform Inversion of Radio Occultation Measurements Made with a Receiver inside the Earth's Atmosphere," Annals of Geophysicae, Vol. 20, No. 8, 2002, pp. 1253-1256. doi:10.5194/angeo-20-1253-2002

[5] R. N. Bracewell and A. C. Riddle, "Inversion of Fan-Beam Scans in Radio Astronomy," Astrophysical Journal, Vol. 150, 1967, pp. 427-434. doi:10.1086/149346

[6] S. C. Solomon, P. B. Hays and V. J. Abreu, "Tomographic Inversion of Satellite Photometry," Applied Optics, Vol. 23, No. 19, 1984, pp. 3409-3414. doi:10.1364/AO.23.003409

[7] E. L. Kosarev, "Applications of Integral Equations of the First Kind in Experiment Physics," Compututer Physics Communications, Vol. 20, No. 1, 1980, pp. 69-75. doi:10.1016/0010-4655(80)90110-1

[8] U. Buck, "Inversion of Molecular Scattering Data," Reviews of Modern Physics, Vol. 46, No. 2, 1974, pp. 369389. doi:10.1103/RevModPhys.46.369

[9] H. Hellsten and L. E. Andersson, "An Inverse Method for the Processing of Synthetic Aperture Radar Data," Inverse Problems, Vol. 3, No. 1, 1987, pp. 111-124. doi:10.1088/0266-5611/3/1/013

[10] R. S. Anderssen and R. B. Calligaro, "Non Destructive Testing of Optical-Fiber Performs," The Journal of the Australian Mathematical Society. Series B. Applied Mathematics, Vol. 23, No. 2, 1981, pp. 127-135. doi: $10.1017 / \mathrm{S} 0334270000000138$ 
[11] K. Taketura, "Index Profile Determination of Single-Mode Fiber by the Phase Contrast Method: A Proposed Technique," Applied Optics, Vol. 21, No. 23, 1982, pp. 42604263. doi:10.1364/AO.21.004260

[12] W. J. Glantschnig, "How Accurately Can One Reconstruct an Index Profile from Transverse Measurement Data," Journal of Lightwave Technology, Vol. 3, No. 3, 1985, pp. 678-683. doi:10.1109/JLT.1985.1074221

[13] E. Keren, E. Bar-Ziv, I. Glatt and O. Kafri, "Measurements of Temperature Distribution of Flames by Moiré Deflectometry," Applied Optics, Vol. 20, No. 24, 1981, pp. 4263 4266. doi:10.1364/AO.20.004263

[14] C. J. Tallents, M. D. J. Burgess and B. Luther-Davies, "The Determination of Electron Density Profiles from Refraction Measurements Obtained Using Holographic Interferometry," Optics Communications, Vol. 44, No. 6, 1983, pp. 384-387. doi:10.1016/0030-4018(83)90222-5

[15] C. Fleurier and J. Chapelle, "Inversion of Abel's Integral Equation Application to Plasma Spectroscopy," Compututer Physics Communications, Vol. 7, No. 4, 1974, pp. 200-206. doi:10.1016/0010-4655(74)90089-7

[16] K. M. Hanson, "Tomographic Reconstruction of Axially Symmetric Objects from a Single Radiograph," Proceed- ings of SPIE 16th International Conference on High Speed Photography and Photonics, Vol. 491, 1984, pp. 180-187.

[17] W. J. Glantschnig and A. Holliday, "Mass Fraction Profiling Based on X-Ray Tomography and Its Application to Characterising Porous Silica Boules," Applied Optics, Vol. 26, No. 6, 1987, pp. 983-989. doi:10.1364/AO.26.000983

[18] A. M. Cormack, "Representation of a Function by Its Line Integrals with Some Radiological Applications," Journal of Applied Physics, Vol. 34, No. 9, 1963, pp. 2722-2727. doi:10.1063/1.1729798

[19] M. Deutsch, A. Notea and D. Pal, "Inversion of Abel's Integral Equation and Its Application to NDT by X-Ray Radiography," NDT International, Vol. 23, No.1, 1990, pp. 32-38.

[20] A. Chakrabarti, "Solution of the Generalized Abel Integral Equation," Journal of Integral Equations and Applications, Vol. 20, No. 1, 2008, pp. 1-11. doi:10.1216/JIE-2008-20-1-1

[21] F. D. Gakhov, "Boundary Value Problems," Pergamon Press, Oxford, 1966. 